\title{
Egressos de um Mestrado Profissional em Saúde da Família: Expectativas, Motivações e Contribuições
}

\author{
Graduates from a Professional Master's \\ Degree Program in Family Health: \\ Expectations, Motivations and Benefits
}

Rocio Fernandes Santos Viniegra ${ }^{\text {I }}$ Luis Guilherme Pessoa da SilvaII Adriana Cavalcanti de AguiarIII Luciana Souza ${ }^{I V}$

\section{PALAVRAS-CHAVE}

- Educação Médica.

- Saúde Pública.

- Avaliação Educacional.

- Avaliação.

- Saúde da Família.

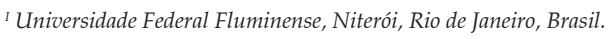

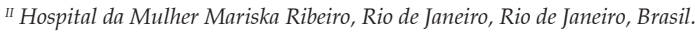

III Fundação Oswaldo Cruz, Rio de Janeiro, Rio de Janeiro, Brasil.

${ }^{I V}$ Universidade do Estado do Rio de Janeiro, Rio de Janeiro, Rio de Janeiro, Brasil. 


\section{KEY-WORDS}

- Medical Education.

- Public Health.

- Educational Measurements.

- Evaluation.

- Family Health.

Recebido em: 19/1/19

Aceito em: 30/3/19

\section{INTRODUÇÃO}

A Estratégia Saúde da Família (ESF), estruturante da atenção primária em saúde, pressupõe a reorganização do modelo assistencial à saúde no Brasil, buscando a superação do modelo hospitalocêntrico, focado no tratamento de doenças. Criada pelo Ministério da Saúde em 1994, sua implantação demanda capacitar profissionais e serviços, com base em princípios pautados na universalidade do acesso, integralidade da atenção e equidade, além de organizar as atividades de forma regionalizada e hierarquizada, dispondo da Unidade Básica de Saúde (UBS) como a principal porta de entrada do sistema ${ }^{1}$.

Cientes da importância dos profissionais como fatores determinantes para o satisfatório funcionamento da ESF e das redes de atenção à saúde, o Ministério da Saúde e o Ministério da Educação definiram mecanismos de incentivo a mudanças na formação, fomentando adequações de currículos de graduação e pós-graduação. Tais mecanismos articularam as Instituições de Ensino Superior (IES) com os serviços de saúde, promovendo a aproximação entre formação de profissionais e produção de conhecimento e a realidade da população brasileira2,3,4.
ABSTRACT

The health care model based on the Family Health Strategy, created in the early 1990s, encouraged changes in health education, highlighting the need to create lato and stricto sensu postgraduate courses aimed at empowering professionals that foster comprehensive health care. Periodic evaluations are carried out and encouraged by Capes/MEC in order to maintain the quality of postgraduate courses, but evaluations of recently-introduced professional master's degree courses in family health remain scarce. Objectives: To describe the academic profile, contribution, motivations and expectations of graduates of a Professional Master's in Family Health. Method: Cross-sectional and quantitative study to analyze the results of 102 questionnaires answered by graduates of the Professional Master's Degree in Family Health of the Estácio de Sá University (RJ), who had concluded the course between 2007 and 2012. The instrument consisted of open-ended and closed-ended questions, sent by e-mail and made available online through the electronic platform Survey Monkey. The study evaluated age, gender, regional origin, academic background, as well as the contributions, expectations and motivations related to the course. Results: The survey sample was formed predominantly by female graduates, aged over 30, from 13 Brazilian states and, mainly from Medicine and Nursing courses. The contribution of the master's degree to the graduate's professional life was evaluated as excellent by $77 \%$ of the interviewees. The expectations regarding the course were positively evaluated and the main reasons for seeking the qualification were scientific-technical improvement and personal satisfaction, rather than better salaries or job stability. Conclusion: The course was evaluated positively by the graduates, having exceeded their expectations and satisfied the interests that led them to it, thus producing changes to their personal and professional life. A longitudinal analysis of the impact of the professional master's degree in the career of graduates will require a sequence of similar studies, as has been stimulated by Capes/MEC in recent years. 
ensino-aprendizagem, com a valorização de metodologias ativas, como também na ressignificação do trabalho fora do ambiente hospitalar, enfatizando a atenção primária e a integração entre os níveis assistenciais, buscando superar a fragmentação do cuidado e a medicalização. Estimula o trabalho em equipe e a formação de vínculo com o usuário de forma individual e familiar, abordando a multicausalidade e a determinação social do processo saúde-doença, demandando competências para atuar na multiplicidade de ações que compõem a ESF ${ }^{6,7}$.

Considerando os profissionais que compõem a equipe de saúde da família, poucos médicos são especialistas nessa área ${ }^{4}$. A fim de preparar e estimular o interesse do médico em trabalhar na atenção primária, a partir de 2014, o Ministério da Educação (MEC) instituiu novas Diretrizes Curriculares Nacionais (DCN) para cursos de Medicina, com a orientação de aproximar escolas e estudantes das redes de serviços de saúde durante a maior parte da graduação. Espera-se, com essa medida, que o estudante adquira formação generalista e humanística, com capacidade de crítica e reflexão, que lhe permita trabalhar em todos os níveis de complexidade da atenção à saúde. Tanto alunos como professores devem ser capazes de exercitar o trabalho em equipe, identificar e refletir sobre problemas da realidade onde está inserido e criar e testar soluções voltadas à sociedade ${ }^{8}$

Tais princípios orientam também o cuidado nos Núcleos de Apoio à Saúde da Família (Nasf), que priorizam o trabalho em equipe na medida em que articulam outros profissionais (fisioterapeutas, nutricionistas, educadores físicos, psicólogos, entre outros) à equipe básica de saúde da família.

O fortalecimento da ESF depende do diálogo entre instituições de ensino, serviços de saúde e comunidades, que deve ser constantemente estimulado por meio da educação permanente, visando ao desenvolvimento de reflexão crítica e melhorias no ambiente e nas relações de trabalho ${ }^{7,9}$.

Nesse sentido, as pós-graduações lato sensu (especialização e residência), voltadas tanto aos recém-formados da graduação quanto aos profissionais já inseridos no SUS, avançaram na formação de quadros para atuarem na saúde da família, focando esforços na articulação do processo de ensino-aprendizagem vinculados aos diversos níveis de complexidade da prática em saúde $\mathrm{e}^{10,11}$. A pós-graduação stricto sensu, desde 1998, conta com a oferta do mestrado profissional, regulamentado pela Coordenação de Aperfeiçoamento de Pessoal de Nível Superior, do Ministério da Educação (Capes), para formação de lideranças para desenvolvimento de tecnologias capazes de dar respostas aos problemas observados na prestação de serviços, aprofundando a interface entre teoria e prática, além de abordar temas relacionados à pesquisa e à docência ${ }^{12}$. Desta forma, tanto a Associação Brasileira de Saúde Coletiva (Abrasco) quanto a Capes consideram o mestrado profissional um adequado formato de qualificação de profissionais para atuarem no SUS, uma vez que favorece a ligação direta do ensino e aprendizagem com o serviço onde o aluno esteja inserido, estimulando a visão crítica da realidade e das experiências ${ }^{7,13}$

Para a Capes,
alguns dos objetivos do mestrado profissional são capacitar profissionais qualificados para o exercício da prática profis- sional avançada e transformadora de procedimentos. Assim como transferir conhecimento para a sociedade, atendendo demandas específicas e de arranjos produtivos para o desen- volvimento nacional, regional ou local. Além de contribuir para agregar competitividade e aumentar a produtividade em empresas, organizações públicas e privadas. ${ }^{14}$

Além de fomentar melhorias nos cursos de graduação e pós-graduação (lato e stricto sensu), o Ministério da Saúde vem estimulando as avaliações contínuas dos processos de trabalho e de ensino-aprendizado das instituições. Estas avaliações, quando realizadas sistematicamente, contribuem para monitorar a qualidade e os resultados dos cursos, criando subsídios para melhorias dos mesmos e de cursos semelhantes ${ }^{6}$

O levantamento bibliográfico demonstrou aumento de estudos sobre avaliação de egressos em formação em saúde no Brasil, porém ainda escassos trabalhos relacionados à pós-graduação em saúde da família, fato também ressaltado por Hortale et al. ${ }^{10}$ e Silva et al. ${ }^{7}$.

Este estudo visa descrever o perfil acadêmico, contribuições, motivações e expectativas de egressos do Mestrado Profissional em Saúde da Família da Universidade Estácio de Sá, no Rio de Janeiro. Assim, colabora com o esforço de avaliação dos cursos de pós-graduação, de forma a estimular a crítica, adequação e consequente melhoria dos currículos e do processo ensino-aprendizagem, na direção de formar um profissional mais preparado para identificar e buscar soluções para as necessidades de saúde da população.

\section{MÉTODOS}

Trata-se de um estudo descritivo, transversal, com abordagem quantitativa, que analisou dados obtidos de questionários respondidos por 102 egressos do curso de Mestrado Profissional em Saúde da Família, da Universidade Estácio de Sá (Unesa), oferecido na cidade do Rio de Janeiro.

Esse curso está inserido na grande área de avaliação da Capes da Saúde Coletiva, tendo iniciado suas atividades em 
2005. Até o ano de 2010, era o único mestrado profissional em Saúde da Família em funcionamento e autorizado pela Capes/MEC no País.

O universo da pesquisa refere-se ao total de 141 alunos que ingressaram no curso nos anos de 2005 a 2010 e que defenderam a dissertação até o mês de fevereiro de 2012.

$\mathrm{O}$ instrumento de pesquisa foi elaborado com base no questionário produzido pela Fiocruz para análise de características dos egressos de seus cursos de doutorado ${ }^{13}$. O questionário elaborado foi pré-testado e corrigido no intuito de aumentar a confiabilidade. Antes de ser enviado ao universo de pesquisa, o questionário revisto foi enviado a cinco egressos do mestrado em análise, em busca de dúvidas sobre o entendimento do conteúdo e tempo de preenchimento. Este foi de aproximadamente 20 minutos, e as dúvidas e sugestões foram analisadas e sanadas.

O questionário utilizado continha 56 questões fechadas e uma aberta e foi dividido em três eixos temáticos. As variáveis pesquisadas na primeira parte relacionavam-se à identificação (idade, sexo, nacionalidade, naturalidade) e à formação acadêmica (ano de ingresso e titulação do mestrado, cursos de graduação e especialização realizados previamente e atividade profissional no ato da matrícula no mestrado). A segunda parte continha variáveis relacionadas à contribuição/impacto do mestrado na vida do egresso. A terceira parte apresentou como variáveis as expectativas e motivações quando da entrada no curso e, por fim, os objetivos/características do curso mais valorizados pelos ex-alunos.

Os egressos foram identificados por meio do banco de dados da secretaria do mestrado, cuja coordenação autorizou a coleta de telefones e $e$-mails para contato. Por ser um curso que atrai estudantes de todo o Brasil e com a finalidade de alcançar a maior parte desses, optou-se por enviar o questionário via e-mail, utilizando-se a plataforma online Survey Monkey (http://www.surveymonkey.com). A análise dos dados obtidos foi realizada com auxílio do software livre Epinfo 7.0.

A coleta de dados foi finalizada em janeiro de 2014, totalizando um período de três meses, nos quais os e-mails foram enviados algumas vezes aos egressos que ainda não haviam respondido ao questionário. A plataforma Survey Monkey foi capaz de identificar os sujeitos da pesquisa que ainda não haviam respondido e garantir o anonimato dos mesmos. Antes do envio do questionário, cada egresso recebeu uma carta-convite, assinada pelo coordenador do curso do mestrado (professor Hésio Cordeiro) e pelo pesquisador, contendo explicação sobre a pesquisa. No corpo do e-mail, seguia um link direcionando para o questionário, que só seria acessado após a concordância com o Termo de Consentimento Livre e Esclare- cido (TCLE). Este esclarecia os objetivos da pesquisa, garantia o anonimato e fornecia dados de contato dos pesquisadores, disponíveis para esclarecer possíveis dúvidas. Caso o sujeito não concordasse com o termo de consentimento, teria a opção de não iniciar o preenchimento.

Esta pesquisa foi analisada e aprovada pelo Comitê de Ética em Pesquisa da Unesa (Protocolo 17472113.5.0000.5284).

\section{RESULTADOS}

Do total de 141 ex-alunos que receberam o diploma de mestrado até fevereiro de 2012 e iniciaram o curso no período de 2005 a 2010, foi possível o contato com 136 egressos, dos quais 102 responderam ao questionário, correspondendo a $72,3 \%$ da população de estudo.

Participaram da pesquisa representantes de 13 estados do Brasil, sobressaindo Rio de Janeiro e Minas Gerais. Predominaram os egressos do sexo feminino $(70,5 \%)$, com idade entre 30 e 39 anos (Tabela 1)

No que concerne à formação acadêmica, observou-se leve predomínio de instituições privadas (53,9\%) sobre as públicas. Os cursos de graduação informados evidenciam a multidisciplinaridade do mestrado em saúde da família, sendo o mais prevalente o de Medicina (35,2\%), seguido por Enfermagem (21,5\%) e Fisioterapia (15,6\%). Ressalta-se que $13,7 \%$ dos egressos concluíram mais de um curso de graduação (Tabela 1).

Quanto à contribuição do mestrado para a vida profissional, 97\% dos egressos a consideraram boa, ótima ou excelente. As justificativas para isto foram solicitadas sob a forma de respostas abertas. Agrupando as respostas positivas em contextos similares, a maioria apontou a ampliação de conhecimentos na área estudada (36\%) e a promoção profissional (20\%). Somente 7\% fizeram referência à contribuição à pesquisa.

Em termos do grau de contribuição do curso nos vários aspectos da vida do egresso, $80 \%$ da amostra analisada revelaram como "alta" a contribuição para a formação técnico-profissional e $60 \%$ mencionaram que houve ampliação das oportunidades de trabalho, havendo também aumento do prestígio profissional (69\%). As respostas relacionadas ao aumento da remuneração (31\%) e à melhora do vínculo empregatício (36\%) foram as menos consideradas pelos entrevistados. No que diz respeito à contribuição do mestrado em aspectos da vida pessoal, observa-se que todos os egressos atribuíram o conceito "alto" à maioria dos quesitos, com ênfase no crescimento pessoal (91\%) (Tabela 2).

As expectativas dos egressos quanto ao Mestrado Profissional em Saúde da Família foram plenamente alcançadas para $66 \%$ dos respondentes, parcialmente alcançadas para $30 \%$, e não satisfatórias para $1 \%$. Os principais motivos que levaram 


\begin{tabular}{|c|c|c|}
\hline $\begin{array}{r}\text { Distribu } \\
\text { o pe }\end{array}$ & $\begin{array}{c}\text { TABELA } 1 \\
\text { o dos egressos } \\
1 \text { sociodemogr }\end{array}$ & ndo \\
\hline & Frequência (N) & Relativo (\%) \\
\hline Sexo & & \\
\hline Feminino & 72 & 70,5 \\
\hline Masculino & 30 & 29,4 \\
\hline Faixas etárias & & \\
\hline 20-29 anos & 20 & 19,8 \\
\hline 30-39 anos & 32 & 31,6 \\
\hline $40-49$ anos & 30 & 29,7 \\
\hline Mais de 50 anos & 17 & 16,8 \\
\hline Origem & & \\
\hline Alagoas & 1 & 0,9 \\
\hline Bahia & 1 & 0,9 \\
\hline Espírito Santo & 5 & 4,9 \\
\hline Goiás & 2 & 1,9 \\
\hline Minas Gerais & 13 & 12,8 \\
\hline Mato Grosso do Sul & 1 & 0,9 \\
\hline Pará & 4 & 3,9 \\
\hline Pernambuco & 1 & 0,9 \\
\hline Piauí & 1 & 0,9 \\
\hline Rio de Janeiro & 67 & 66,3 \\
\hline Rio Grande do Norte & 1 & 0,9 \\
\hline Roraima & 1 & 0,9 \\
\hline São Paulo & 3 & 2,9 \\
\hline Angola & 1 & 0,9 \\
\hline Formação acadêmica & & \\
\hline Instituição privada & 55 & 53,9 \\
\hline Instituição pública & 47 & 46,0 \\
\hline Curso de graduação & & \\
\hline Medicina & 36 & 35,2 \\
\hline Enfermagem & 22 & 21,5 \\
\hline Fisioterapia & 16 & 15,6 \\
\hline Odontologia & 6 & 5,8 \\
\hline Farmacologia & 6 & 5,8 \\
\hline Psicologia & 5 & 4,9 \\
\hline Biologia & 5 & 4,9 \\
\hline Nutrição & 2 & 1,9 \\
\hline Outros & 4 & 3,9 \\
\hline
\end{tabular}

os alunos a buscar o curso foram a busca de aprimoramento técnico-científico (87\%), melhora da satisfação pessoal (82\%) e evolução da carreira docente (73\%). Foi identificado menor interesse quanto à visibilidade do curso (19\%) e à recomendação institucional (28\%) (Tabela 3).

\begin{tabular}{|c|c|c|c|}
\hline \multicolumn{4}{|c|}{$\begin{array}{c}\text { TABELA } 2 \\
\text { Contribuição do mestrado em aspectos da } \\
\text { vida profissional e pessoal do egresso }\end{array}$} \\
\hline & $\begin{array}{l}\text { Baixa } \\
(\%)\end{array}$ & $\begin{array}{l}\text { Média } \\
(\%)\end{array}$ & $\begin{array}{l}\text { Alta } \\
(\%)\end{array}$ \\
\hline \multicolumn{4}{|l|}{ Contribuição para a vida profissional } \\
\hline Formação técnico-profissional & 7 & 13 & 80 \\
\hline $\begin{array}{l}\text { Ampliação das oportunidades de } \\
\text { trabalho }\end{array}$ & 15 & 24 & 60 \\
\hline Aumento da remuneração & 31 & 34 & 33 \\
\hline Aumento do prestígio profissional & 5 & 24 & 69 \\
\hline Melhora do vínculo empregatício & 36 & 25 & 37 \\
\hline \multicolumn{4}{|l|}{ Contribuição para a vida pessoal } \\
\hline Crescimento pessoal & 0 & 8 & 91 \\
\hline $\begin{array}{l}\text { Criação ou ampliação de redes de } \\
\text { relações }\end{array}$ & 8 & 26 & 65 \\
\hline Aumento de autonomia no trabalho & 25 & 24 & 48 \\
\hline Melhora nas relações de trabalho & 20 & 30 & 49 \\
\hline Melhora na oratória & 17 & 18 & 65 \\
\hline Melhora na vida pessoal/social & 17 & 24 & 56 \\
\hline
\end{tabular}

\begin{tabular}{|c|c|c|}
\hline $\begin{array}{r}\text { TABELA } \\
\text { Motivos para busca } \\
\text { Profissional em Saú }\end{array}$ & $\begin{array}{l}\text { Estrado } \\
\text { Família }\end{array}$ & \\
\hline Motivos & $\begin{array}{l}\text { Absoluto } \\
\text { (N) }\end{array}$ & $\begin{array}{c}\text { Relativo } \\
(\%)\end{array}$ \\
\hline Aprimoramento técnico-científico & 89 & 87,2 \\
\hline Satisfação pessoal & 84 & 82,3 \\
\hline Evolução da carreira docente & 75 & 73,5 \\
\hline Reciclagem de conhecimento & 47 & 46,0 \\
\hline Aumento de salário & 36 & 35,2 \\
\hline Oportunidade de emprego & 34 & 33,6 \\
\hline $\begin{array}{l}\text { Recomendação institucional, focando } \\
\text { aprimoramento profissional }\end{array}$ & 28 & 27,4 \\
\hline Visibilidade do curso & 19 & 18,6 \\
\hline
\end{tabular}

Elencados os principais objetivos do curso de mestrado em apreço, foi solicitado que os egressos apontassem os considerados relevantes durante seu percurso de aprendizado. "Conhecimento/domínio de ferramentas de diagnóstico e avaliação de problemas na atenção básica" foi o mais cotado (80\%), seguido por "conhecimento sobre importância e complexidade da ESF" (78\%) e por "conhecimento sobre processo de cuidado à saúde e sobre a gestão da atenção básica no SUS" (72\%). Os menos cotados foram "desenvolvimento de projeto científico relevante para Saúde Coletiva" e "conhecimento sobre a implantação da ESF", ambos com 59\% (Tabela 4). 


\begin{tabular}{|c|c|c|}
\hline $\begin{array}{l}\text { TABELA } 4 \\
\text { Considerações relevante } \\
\text { objetivos do curso de me }\end{array}$ & $\begin{array}{l}\text { sobre } \\
\text { strado }\end{array}$ & \\
\hline Considerações sobre o curso & $\begin{array}{l}\text { Absoluto } \\
\text { (N) }\end{array}$ & $\begin{array}{c}\text { Relativo } \\
(\%)\end{array}$ \\
\hline $\begin{array}{l}\text { Conhecimento/domínio de ferramentas de } \\
\text { diagnóstico e avaliação de problemas na } \\
\text { atenção básica }\end{array}$ & 82 & 80,3 \\
\hline $\begin{array}{l}\text { Conhecimento sobre importância e } \\
\text { complexidade da ESF }\end{array}$ & 80 & 78,4 \\
\hline $\begin{array}{l}\text { Conhecimento sobre processo de cuidado } \\
\text { à saúde e sobre a gestão da atenção básica } \\
\text { no SUS }\end{array}$ & 73 & 72,2 \\
\hline $\begin{array}{l}\text { Conhecimento relacionado à docência } \\
\text { com estímulo a mudanças na formação em } \\
\text { saúde e produção de conhecimento }\end{array}$ & 65 & 63,7 \\
\hline $\begin{array}{l}\text { Melhora das relações interpessoais e } \\
\text { contatos profissionais }\end{array}$ & 60 & 58,8 \\
\hline Conhecimento sobre a implantação da ESF & 59 & 57,8 \\
\hline $\begin{array}{l}\text { Desenvolvimento de projeto científico } \\
\text { relevante para Saúde Coletiva }\end{array}$ & 59 & 57,8 \\
\hline
\end{tabular}

\section{DISCUSSÃO}

No Brasil, a Capes é a instância que avalia cursos stricto sensu no sentido de manter ou aumentar a qualidade e adequá-los às exigências cabíveis. Avaliar um curso de pós-graduação é um processo complexo e delicado, pois expõe seu desempenho e sua imagem, além de interesses políticos e ideológicos. Ao mesmo tempo, esse processo detecta pontos positivos, fragilidades e cria subsídios para seu aprimoramento. A análise de egressos realizada pela instituição de ensino contribui com informações sobre o impacto do curso na vida do aluno e na sociedade, identificando dificuldades e êxitos ${ }^{6,14}$.

O número de respondentes (102) corresponde a $72 \%$ do universo de pesquisa (141), superior à taxa de respostas observadas na literatura para estudos que utilizam o $e$-mail como meio de acesso aos egressos. Acredita-se que a carta-convite assinada pelo coordenador do curso, professor Hésio Cordeiro, conferiu confiabilidade e importância ao $e$-mail e à pesquisa. Apesar de os questionários online assumirem importante espaço na investigação científica como alternativa aos métodos tradicionais (papel), questiona-se a baixa utilização e adesão dos respondentes, possivelmente justificada pela percepção de um e-mail não desejado, impessoalidade do contato, dificuldade em utilizar informática ou o desenho de estudo não adequado ${ }^{15}$. O reenvio persistente do questionário por determinado período não garante aumento na taxa de respostas capazes de gerar significância estatística ${ }^{16}$.

Houve predomínio do sexo feminino (70\%), de modo análogo a outras publicações nacionais sobre avaliação de egressos em saúde, tanto de cursos lato quanto stricto sensu ${ }^{6,13,17,18} \mathrm{O}$ predomínio do grupo etário de 30 a 39 anos, com considerável número de respondentes acima dos 40 anos, provavelmente justifica-se por se tratar de um curso pioneiro no País, atendendo a uma demanda reprimida de profissionais interessados nessa área do conhecimento, fato corroborado pela presença de alunos das cinco regiões do País, principalmente nos anos iniciais de oferta do curso. Como esperado, pela proximidade, há predomínio de alunos da Região Sudeste, predominando o Estado do Rio de Janeiro ${ }^{7,13,17}$

Mudanças nos processos de trabalho exigidas pelo SUS, associadas à ampliação da ESF, cobraram do governo e dos profissionais novas competências. Embora os esforços para criar políticas de ensino voltadas aos profissionais atuantes no SUS existam há mais de 15 anos, ainda em 2018, Cavalli e Rizzotto ${ }^{4}$, ao analisarem a formação de médicos que atuam como líderes da Atenção Primária em Saúde (APS) no Paraná, identificaram falta de conhecimento direcionado ou considerado suficiente para trabalhar na ESF. Torna-se evidente a insatisfação pessoal e profissional, além da necessidade de complementar a educação direcionada à área em questão. Entre as formas de educação apontadas (especializações, mestrados, residência), as especializações acabam sendo mais procuradas, e, apesar das críticas (são mais teóricas que práticas e têm carga horária reduzida), são consideradas importantes para melhorar a assistência e a implantação da ESF no País.

A Política Nacional de Educação Permanente em Saúde (PNEPS), de 2004, estipula transformações nos processos de ensino em saúde prioritariamente voltados ao SUS, articulando e fortalecendo o ensino, serviço e comunidade de forma a identificar e resolver problemas do território onde o aluno ou profissional está inserido, enfocando o cotidiano de trabalho como campo de estudo e aprendizado ativo ${ }^{7,19,20}$.

O Mestrado Profissional em Saúde da Família oferece oportunidades de problematização preconizadas na PNEPS e obteve projeção nacional, antecedendo cursos com objetivos análogos, como o da Rede Nordeste de Formação em Saúde da Família (Renasf), iniciado em $2012^{22}$.

Em relação à formação acadêmica dos participantes, predominaram as instituições privadas, prevalecendo as profissões de médicos, seguidas por enfermeiros e fisioterapeutas. Considerando que médicos e enfermeiros são os profissionais mais frequentes nas equipes de Saúde da Família, esperava-se que fossem as graduações mais citadas. Maciel et al. ${ }^{6} \mathrm{e}$ Melo ${ }^{22}$ observaram como formações acadêmicas dominantes a de enfermeiros, odontólogos e médicos quando da avaliação de especializações e residência multiprofissional em Saúde da Família, assim como do Mestrado Profissional em Saúde da Família da Renasf. 
A demanda pelo curso por profissionais como fisioterapeutas, odontólogos e educadores físicos, entre outros, é compatível com a criação dos Núcleos de Apoio a Saúde da Família (Nasf), em 2008, com o intuito de solucionar problemas por meio do trabalho em equipe multidisciplinar, matriciamento e compartilhamento de saberes, experiências, habilidades e responsabilidades ${ }^{9,22}$. Ao mesmo tempo, a diversidade de formações acadêmicas, somada às áreas de atuação dos profissionais (como gestão, docência e assistência), enriquece a operacionalização do currículo do curso ${ }^{22}$

A contribuição do mestrado na vida do egresso foi considerada ótima ou excelente, e apenas cerca de 3\% responderam que não houve contribuição ou houve decepção. Sobre a contribuição na vida profissional, destacam-se a formação técnica, o prestígio profissional e a ampliação das oportunidades de trabalho. Já na contribuição na vida pessoal, destacam-se o crescimento pessoal, melhoras na oratória e criação ou ampliação das redes de relações. Fatores similares foram observados no estudo de Gomes e Goldemberg ${ }^{18}$ na análise de egressos de pós-graduação stricto sensu (doutorado, mestrado profissional e acadêmico) na área da Saúde Coletiva.

Todos os estudos que investigaram egressos de cursos voltados à saúde da família confirmaram contribuições positivas para a vida do discente, ressaltando melhora no planejamento de ações a partir da análise epidemiológica local, possibilitando melhores diagnósticos locais, ampliação da visão da saúde, capacidade de solucionar problemas, melhorias nos processos de trabalho e conhecimentos sobre gerência, com aplicabilidade em seu cotidiano, resultando em serviços de maior qualidade ofertados na ESF ${ }^{6,7,13,22}$ Em consonância, para Ramos et al. ${ }^{12}$, a contribuição ideal esperada de um mestrado profissional na área da saúde seria a construção de uma pesquisa inovadora, bem elaborada e divulgada, capaz de trazer ao seu autor benefícios não só acadêmicos como de reconhecimento profissional e pessoal, à medida que articulasse a produção de conhecimento com sua aplicação na prática dos serviços e sistemas de saúde. Ramos et al. ${ }^{12}$ também percebem e questionam o distanciamento entre os serviços de saúde e de ensino, o que gera decepção ao egresso quando este entende a dificuldade de aplicar na prática os novos conhecimentos, o que autentica o valor das avaliações internas dos programas e processos de ensino.

Neste estudo, não foram explicitados pontos negativos, o que minimiza seu potencial de informar estratégias para seu aprimoramento. Contudo, avaliações de egressos de outros cursos em saúde da família destacam como ponto de fragilidade a falta de articulação do aprendizado com a prática do SUS, principalmente para alguns participantes que não trabalham ou não se identificam com a ESF, contrariando os investi- mentos e orientações do Mistério da Saúde ${ }^{6,7,12}$. Outros pontos de debilidades identificados foram a falta de disciplinas de didática ${ }^{17}$, dificuldades na elaboração do projeto, a realização de pesquisas e o contato com metodologias ativas de ensino-aprendizagem, reconhecidas como novas e desafiadoras para muitos alunos 22

Inversamente, os discentes deste estudo apontaram como contribuição o auxílio na evolução da carreira docente, mesmo que metade dos egressos já trabalhassem nesta área, demonstrando o impacto da pós-graduação stricto sensu na formação dos professores ${ }^{14}$

Remuneração e vínculo empregatício foram os quesitos que menos contribuíram para a vida de dois terços dos egressos, indicando que o diploma de mestrado não é garantia de acréscimo nesses aspectos importantes da atuação profissional, podendo gerar decepção para alguns. Gomes e Goldemberg $^{18}$, em estudo pós-conclusão de cursos stricto sensu em Saúde Coletiva, observaram frequente descontentamento com a remuneração auferida.

Um terço dos egressos do presente estudo, por outro lado, julgou positivamente a melhora do vínculo de trabalho e aumento de salário, o que pode corresponder à progressão funcional daqueles inseridos formalmente em atividades de docência universitária. O mesmo foi observado por Mendes et al. ${ }^{17}$ e Ferreira e Morraye ${ }^{23}$ ao avaliarem egressos de mestrados em Ciências e Saúde e em Promoção de Saúde, respectivamente, e acrescentam a ideia de que, quanto maior o acúmulo de anos de estudo, maior a remuneração.

Sabe-se que a precariedade de vínculos e a remuneração são importantes causas de rotatividade entre os profissionais da ESF, principalmente os médicos. Durante a organização do SUS e da ESF, o governo federal atribuiu às prefeituras municipais a responsabilidade dos contratos com os trabalhadores da saúde. A escassez de concursos públicos é concomitante à terceirização mediante cooperativas, com poucos direitos trabalhistas, culminando em vínculos enfraquecidos e migrações em busca de melhores condições ou rendas complementares ${ }^{21}$. Isto foi observado por Gonçalves et al..$^{5}$ em seu estudo com 20 equipes do município de Montes Claros (MG), onde 97\% dos profissionais trabalham com contratos temporários.

Possíveis soluções devem ser examinadas com vistas à valorização dos vínculos trabalhistas a fim de favorecer a permanência dos profissionais de saúde nas equipes da atenção básica: organização de concursos públicos, planos de cargos e salários, estímulo à qualificação e educação permanente e gestão participativa ${ }^{24}$

Em relação aos motivos apontados pelos egressos para a busca do curso em questão, os mais relevantes foram aprimo- 
ramento técnico-científico, satisfação pessoal e evolução da carreira docente. Hortale et al. ${ }^{10}$ encontraram como motivações para busca do mestrado profissional para servidores da Fiocruz a iniciativa pessoal e a recomendação institucional como forma de aprimoramento profissional.

Neste estudo, mais da metade dos egressos refere como motivo de busca do mestrado a "evolução da carreira docente", e quase um terço considera "aumento de salário" e "recomendação institucional", levando a refletir que o corpo discente foi composto por profissionais atuantes na docência e/ou na ESF. Ao associar a faixa etária dos egressos - quase a metade acima de 40 anos - com os motivos da busca do mestrado, ponderam-se interesses financeiros relacionados à evolução da carreira e ao aprimoramento do conhecimento por profissionais que levam tempo inseridos nos serviços de saúde e educação.

O interesse pelo mestrado profissional, fomentado ou não pelo empregador, ocorre em profissionais da saúde cujos currículos são anteriores ou posteriores às novas DCN, mas que sentem necessidade de expandir conhecimentos nas questões práticas e cotidianas das profissões, associando atualizações das competências tecnológicas e das necessidades sociais ${ }^{4}$

As expectativas dos egressos em relação ao curso foram plenamente alcançadas por dois terços dos respondentes, assim como observado por Ferreira e Morraye ${ }^{24}$, que encontraram satisfação em $62,8 \%$ das avaliações de egressos de pós-graduação em promoção da saúde. Não houve, porém, detalhamento sobre a avaliação das expectativas.

Em concordância com os objetivos do mestrado profissional determinados pela Capes, os egressos informaram melhorias no conhecimento de habilidades voltadas à prática diária de trabalho, conhecimento sobre a importância e complexidade da ESF, sobre o processo de cuidado à saúde e gestão da atenção básica, temas análogos aos observados por Maciel et al. ${ }^{6}$ e Santos ${ }^{20}$

Os conhecimentos analisados se relacionam às competências necessárias a uma boa qualidade do trabalho em saúde da família. Estas, por sua vez, apresentam características complexas e dinâmicas, com frequência incorporam novas tecnologias, novos saberes, novas formas de ensino-aprendizagem e requerem novas atitudes dos profissionais ${ }^{5}$. Em consonância com a Capes, o mestrado profissional associa teoria e prática nos processos de aprendizado, visando capacitar profissionais orientados a atender às demandas sociais ${ }^{14}$.

Estima-se, assim, a importância dos cursos de pós-graduação lato e stricto sensu como estratégias complementares de qualificação profissional capacitadoras de quadros para atuarem na ESF. O mestrado profissional em questão apresenta características sobrepostas de educação, pautadas na atualização e aprofundamento técnico voltado à saúde da família, assim como traz problematizações das situações identificadas nos serviços do SUS, onde atuam seus estudantes. Pretende-se estimular a análise de um problema identificado no local de trabalho, relacionando-o com seus determinantes sociais e econômicos, e explorar soluções por meio da abordagem crítica desses fatores. Espera-se instigar a reflexão sobre o processo de trabalho, transformando o profissional em responsável pelos procedimentos de ensino e aprendizagem. Desta forma, o curso em análise apresenta também características e valores da educação permanente, contribuindo com a reorganização dos modelos assistenciais e respondendo às recomendações das políticas educacionais do setor da saúde $e^{5,20}$.

\section{CONSIDERAÇÕES FINAIS}

Os resultados da pesquisa revelam egressos do Mestrado Profissional em Saúde da Família da Unesa predominantemente do sexo feminino, maiores de 30 anos, oriundos de vários estados do Brasil, principalmente do Rio de Janeiro. As profissões mais frequentes foram as de médicos, enfermeiros e fisioterapeutas.

O curso em análise, por atrair alunos que provavelmente já trabalhavam com saúde pública, influenciou favoravelmente a vida do egresso em níveis diversos de aproveitamento prático e teórico. Trata-se de um período de crescimento pessoal e profissional importante, em que ocorre a consolidação de conhecimentos e reflexões críticas sobre a área de atuação, contribuindo para a formação de quadros mais preparados para os desafios de um sistema complexo e todavia carente de profissionais capacitados.

O aumento da oferta e da procura por mestrados profissionais na área da Saúde da Família e Saúde Coletiva indica a necessidade de aprimoramento profissional, de evolução dos processos de ensino e de preocupação com avaliações. Os cursos podem internalizar os valores da educação permanente, como estímulo ao aprendizado contínuo, exercício de novos conceitos e problematização dos processos de trabalho. Nesse sentido, percebeu-se a importância desse curso para o aperfeiçoamento profissional e pessoal do egresso, constituindo uma valiosa ferramenta de ensino. A recente aprovação, pela Capes, da proposta de doutorado profissional em saúde da família, encaminhada pelo grupo de docentes do curso aqui analisado, reitera o acerto das opções teórico-metodológicas do mestrado e antecipa novos desafios.

Fato observado em muitos trabalhos de avaliação de cursos e egressos, e este não fugiu à regra, foi a desatualização de dados cadastrais e a consequente dificuldade de acesso aos 
sujeitos da pesquisa, além do acompanhamento dos mesmos pela instituição de ensino.

Espera-se que a divulgação deste resultado possa subsidiar e incentivar este e outros cursos a incluírem e manterem a prática avaliativa, fundamental à evolução e ao aprimoramento da formação de recursos humanos adequados a um sistema de saúde mais humanizado e resolutivo integralmente.

\section{REFERÊNCIAS}

1. Jesus RL, Engstrom E, Brandão AL. A expansão da Estratégia Saúde da Família no Rio de Janeiro, 2009-2012: estudo de caso numa área da cidade. Rev Bras Med Fam Comunidade. [online]. 2015. 10(37) [capturado em maio 2017]; 1-11. Disponível em: https:/ / rbmfc.org.br/rbmfc/article/ view $/ 975$

2. Brasil. Decreto $n^{\circ} 7.508$, de junho de 2011. Regulamenta a Lei no 8.080, de 19 de setembro de 1990, para dispor sobre a organização do Sistema Único de Saúde - SUS, o planejamento da saúde, a assistência à saúde e a articulação interfederativa, e dá outras providências. [online]. 2011 [capturado em julho 2017] Disponível em: http: / / www.planalto. gov.br/ccivil_03/_ato2011-2014/2011/decreto/d7508.htm

3. Brasil. Lei $n^{\circ} 12.871$, de 22 de outubro de 2013. Institui o Programa Mais Médicos. [online]. 2013 [capturado em julho 2017] Disponível em: http:/ / www.planalto.gov.br / ccivil_03/_ato2011-2014/2013/lei/112871.htm

4. Cavalli, LO; Rizzotto, MLF. Formação dos Médicos que Atuam como Líderes das Equipes de Atenção Primária em Saúde no Paraná. Rev. Bras. Educ. Med. vol.42 no.1 Brasília Jan./Mar. 2018

5. Gonçalves CR, Cruz MT, Oliveira MP, Morais JAD, Moreira KS, Rodrigues ACQ, Leite MTS. Recursos humanos: fator crítico para as redes de atenção à saúde. Saúde em Debate 2014; vol. 38, núm. 100, 26-34.

6. Maciel ELN, Figueiredo PF, Prado RN, Galavote HS, Ramos MC, Araujo MD, Lima RCD. Avaliação dos egressos do curso de especialização em Saúde da Família no Espírito Santo. Ciência e Saúde Coletiva. 2010; 15(4):2021-2028.

7. Silva TAM, Fracolli LA, Chiesa AM. Trajetória Profissional na estratégia Saúde da Família: em Foco a Contribuição dos Cursos de Especialização. Rev. Latino-Am. Enfermagem [online]. 2011. 19(1) [capturado maio 2017]; 8 telas. Disponível em: http://www.scielo.br/pdf/rlae/v19n1/ pt_20.pdf

8. Brasil, Ministério da Educação. Conselho Nacional de Educação. Câmara de Educação Superior. Resolução CNE/CES n³ de 20 de junho de 2014. Institui Diretrizes Curriculares Nacionais do Curso de Graduação em Medi- cina. Diário Oficial da União. Brasília, 23 de junho de 2014; Seção 1, p. 8-11.

9. Brasil. Ministério da Saúde. Secretaria de Atenção à Saúde. Departamento de Atenção Básica. Diretrizes do NASF: Núcleo de Apoio a Saúde da Família. Brasília: Ministério da Saúde, 2010. [online] 2010 [Acessado em julho 2017]; p.7-154 Disponível em: http://bvsms.saude.gov.br/bvs/ publicacoes/caderno_atencao_basica_diretriz es_nasf.pdf

10. Hortale VA, Dias MAS, et al. Construção Teórico-Metodológica e Aprendizados com a Experiência no Mestrado Profissional em Saúde da Família. TrabEduc.Saúde [online]. 2015. v.13, supl.2, [capturado em maio 2017]; p.11-23. Disponível em: http:/ /dx.doi.org/10.1590/1981-7746-sip00078

11. Engstrom EM, Motta JI, Venancio SA. A formação de profissionais na pós-graduação em saúde pública e atenção primária à saúde no município do Rio de Janeiro, Brasil. Ciência \& Saúde Coletiva 2016; 21(5):1461-1470.

12. Ramos F, Backes V, Backes D, Schneider D, Pinheiro G, Zeferino M, Rocha P. Formação de mestres em enfermagem na Universidade Federal de Santa Catarina: contribuições sob a ótica de egressos. Rev. Bras. Enferm [online]. 2010.v. 63, n. 3, [capturado em jan 2016]. Disponível em: http:/ / www.scielo.br/scielo.php?script=sci_arttext\&pid =S0034-71672010000300002

13. Hortale VA, Leal MC, Moreira COF, Aguiar AC. Características e limites do mestrado profissional na área da Saúde:estudo com egressos da Fundação Oswaldo Cruz. Ciência e Saúde Coletiva 2010; 15(4):2051-2058

14. CAPES - Coordenação de Aperfeiçoamento de Pessoal de Nível Superior. [online] 2014 [capturado em janeiro 2014] Disponível em: http://www.periodicos.capes.gov.br/index.php?option $=$ com_pcontent $\& v i e w=$ pcontent $\&$ alias $=m$ issao-objetivos\&Itemid $=102$

15. Terrível J, Rodrigues AT, Ferreira M, Neves C, Roque F, Silva OABC, Figueiras A, Herdeiro MT.Conhecimento dos médicos relativo à prescrição de antibióticos à resistência antimicrobiana: Estudo piloto de comparação de questionário online vs papel. RevEpidemiolControlInfect. 2013;3(3):93-98

16. Willis GB, Smith T, Lee JH. Do Additional Recontacts to Increase Response Rate Improve Physician Survey Data Quality?MedCare. [online] 2013 Oct; 51(10): 945-948. Capturado em 19 de dez. de 2018. Disponível em: https:/ / www.ncbi.nlm.nih.gov/pmc/articles/PMC3784997/

17. Mendes RF, Venceslau EOO, Aires AS, Junior RRP. Percepção sobre o curso e perfil de egressos do Programa de Mestrado em Ciências e Saúde da UFPI. RBPG. 2010; v. 7, n. 12 , p. 82-101. 18 
18. Gomes MHA, Goldemberg P. Retrato quase sem retoques dos egressos dos programas de pós-graduação em Saúde Coletiva, 1998-2007. Ciência e Saúde Coletiva 2010; 15(4):1989-2005.

19. Brasil. Ministério da Saúde (MS). Portaria GM/MS nº 198, de 13 de fevereiro de 2004. Institui a Política Nacional de Educação Permanente em Saúde como estratégia do Sistema Único de Saúde para a formação e o desenvolvimento de trabalhadores para o setor dá outras providências. Diário Oficial da União. Brasília. Portaria nº 198/GM em 13 de fev. de 2004.

20. Santos, BOA. Formação dos profissionais de saúde que atuam na Estratégia de Saúde da Família do Município de Caucaia (CE). Ceará; 2016. Mestrado [Dissertação] - Universidade Federal do Ceará. Faculdade de Medicina. (Mestrado Profissional em Saúde da Família)

21. Medeiros CRG, Junqueira AGW, Schwingel G, Carreno I, Jungles LAP, Saldanha MFL. A rotatividade de enfermeiros e médicos: um impasse na implementação da Estratégia de Saúde da Família. Ciência \& Saúde Coletiva 2010; 15(Supl. 1):1521-1531

22. Melo, NSMN. Mestrado profissional em saúde da família: o curso sob a perspectiva dos mestrandos. Rio Grande do Norte (Natal); 2014. Mestrado [Dissertação] - Centro de Ciências da Saúde, Universidade Federal do Rio Grande do Norte (Mestrado Profissional em Saúde da Família)

23. Ferreira, RS; Morraye, MA. Perfil dos mestres de um programa de pós-graduação em Promoção de Saúde: características e percepções sobre o curso. RBPG, Brasília, v. 10, n. 22, p. 1085 - 1107, dezembro de 2013.

24. Ney M; Rodrigues PHA. Fatores Críticos para fixação do médico na ESF. Physis [online]. 2012. vol.22 no.4 [capturado em dez. 2018] Disponível em: http://dx.doi. org/10.1590/S0103-73312012000400003

\section{CONTRIBUIÇÃO DOS AUTORES}

Rocio Fernandez Santos Viniegra participou de todas as etapas do desenvolvimento deste manuscrito, incluindo o desenho do estudo, revisão da literatura, adaptação do questionário, coleta de dados, análise, elaboração da versão inicial e final do artigo. Luiz Guilherme Pessoa da Silva participou da elaboração do desenho do estudo, adaptação do questionário, análise, elaboração da versão inicial e final do artigo. Adriana Cavalcanti de Aguiar participou do desenho de estudo, contribuindo substancialmente com a revisão crítica do texto e elaboração da versão final. Luciana Maria Borges da Matta Souza contribuiu com a revisão crítica do texto.

\section{CONFLITO DE INTERESSES}

Os autores declaram não haver conflito de interesses.

\section{ENDEREÇO PARA CORRESPONDÊNCIA}

Rocio Fernandez Santos Viniegra. Avenida Rui Barbosa 40/2002, Flamengo, CEP: 22250020; Rio de Janeiro, RJ, Brasil. E-mail: rocioviniegra2015@gmail.com 